\begin{tabular}{l} 
JOURNAL Of \\
Physical Activity \\
\& Health \\
\hline
\end{tabular}

\title{
RELATIONSHIP BETWEEN METABOLIC SYNDROME AND MODERATE-TO-VIGOROUS PHYSICAL ACTIVITY IN YOUTH
}

\begin{tabular}{|r|l|}
\hline Journal: & Journal of Physical Activity \& Health \\
\hline Manuscript ID: & JPAH_2013_0053.R1 \\
\hline Manuscript Type: & Article \\
\hline Keywords: & metabolism, physical activity, health behavior, youth \\
\hline \multicolumn{2}{|c}{} \\
\hline
\end{tabular}

\footnotetext{
SCHOLARONE ${ }^{\text {M }}$

Manuscripts
} 


\section{RELATIONSHIP BETWEEN METABOLIC SYNDROME AND MODERATE-} TO-VIGOROUS PHYSICAL ACTIVITY IN YOUTH

9 Journal: Journal of Physical Activity and Health

Manuscript Type: Original research

12

Running head: Metabolic and behavioral risk factors in youth

Number of words: 5085 (including abstract, references, tables, and acknowledgment)

\section{Number of table: 3}

Abstract word count: 214

20

21

Keywords: Metabolism, Physical Activity, Health Behaviour, Youth

22

Declaration of interest: The authors report no conflicts of interest.

Date of manuscript re-submission: September $24^{\text {th }}, 2013$ 
Metabolic and behavioral risk factors in youth

\section{ABSTRACT}

3

4 Background: Associations of metabolic syndrome (MetS) with lifestyle behaviours in 5 youth is potentially important for identifying subgroups at risk and encourage

6 interventions. This study evaluates the associations among the clustering of metabolic

7 risk factors and moderate-to-vigorous physical activity (MVPA) in youth. Methods:

8 The sample comprised 924 youth (522 girls) aged 11-17 years. Height, weight, waist

9 circumference (WC), fasting glucose, HDL-cholesterol, triglycerides, and blood

10 pressures were measured. Cardiorespiratory fitness (CRF) was assessed using the 20-m

11 shuttle run test. MVPA was estimated with a 3-day diary. Outcome variables were

12 statistically normalized and expressed as $Z$ scores. A clustered metabolic risk score was

13 computed as the mean of $Z$ scores. Multiple linear regression was used to test

14 associations between metabolic risk and MVPA by sex, adjusted for age, WC and CRF.

15 Results: After adjustment for potential confounders, MVPA was inversely associated

16 with the clustering of metabolic risk factors in girls, but not in boys; in addition, after

17 adjusting for $\mathrm{WC}$, the statistical model of that relationship was substantially improved

18 in female youth. Conclusion: MVPA was independently associated with increased risk

19 of MetS in girls. Additional efforts are needed to encourage research with different

20 analytical approach and standardization of criteria for MetS in youth.

21

22 Keywords: Metabolism, Moderate-to-Vigorous Physical Activity, Inactivity, Youth 
Metabolic and behavioral risk factors in youth

1 INTRODUCTION

2 The Metabolic Syndrome (MetS) is often defined as the clustering of three or more risk

3 factors including adiposity, hypertension, hyperglycemia, low high-density lipoprotein-

4 cholesterol (HDL-C), and high triglycerides ${ }^{1}$. The prevalence of MetS has increased

5 among youth and is an increasingly important health challenge worldwide ${ }^{2}$. Based on

6 modified ATPIII criteria ${ }^{3}$, prevalence estimates of MetS among obese adolescents

7 range from $18 \%$ in Spain ${ }^{4}$ to $42 \%$ in the US ${ }^{5}$. The most common metabolic

8 abnormalities among youth with MetS were elevated waist circumference (WC, 96.2\%),

9 low HDL-C (96.2\%) and hypertriglyceridemia (73.1\%); insulin resistance was also

10 identified in youth having MetS ${ }^{6}$. Relatively recent environmental and behavioural

11 changes associated with increased sedentary behaviour and reduced physical activity

12 (PA) may have contributed to this phenomenon.

13 Health authorities in most countries have recognized the potentially negative

14 effects of inactive lifestyles for health and have compiled guidelines to enhance the 15 level of PA among children and adolescents ${ }^{7}$. Recent reviews confirm the importance 16 of improving habitual PA in youth and suggest that higher levels of moderate to17 vigorous physical activity (MVPA) are inversely associated with adiposity ${ }^{8}$ and an 18 adverse cardiometabolic risk profile ${ }^{9-11}$. However, with an increase in MVPA, the risk 19 of an unfavourable risk profile is reduced ${ }^{2,10}$.

20 Adolescence is a period during which involvement in PA may contribute to a 21 physically active lifestyle that persists into adulthood ${ }^{12}$. Young people, particularly 22 during adolescence, tend to show lower levels of PA and should be a target for 23 prevention strategies aimed at healthy lifestyles. For example, $80 \%$ or more of 24 adolescent girls in 100 of 105 countries (95\%) and of adolescent boys in $56(53 \%)$ of 25105 countries did not achieve the objective of 60 minutes MVPA per day ${ }^{13}$. As such, 
1 better understanding of interactions among MVPA and metabolic health of youth,

2 particularly in under studied populations of children and adolescents, can be helpful in

3 designing effective and targeted strategies to reduce metabolic disease risk.

4 In context of the preceding trends, the objective of this study is to evaluate the

5 relationships between the clustering of metabolic risk factors and MVPA among

6 adolescents aged 11-17 years after adjusting for several potential confounders. It was

7 hypothesized that adolescents classified as less active would be more likely to have

8 higher metabolic risk than more active peers.

\section{METHODS}

\section{Sample}

12 The cross-sectional study was carried out in Curitiba (about 1,678,965 inhabitants), 13 Paraná, Brazil. Curitiba has nine administrative districts with 293 schools. The 14 proportion of students in each of the nine administrative areas was as follows: Santa 15 Felicidade 6.6\%; Matriz, 12.3\%; Boa Vista, 14.7\%; Cajuru, 12\%; Portão, 10.6\%; 16 Boqueirão, 13.1\%; Bairro Novo, 9.6\%; Pinheirinho, 9.5\%; and CIC, 11.6\%. Schools 17 were randomly selected among the districts and all students in the respective schools 18 were invited to participate in the study. The final sample represents the students who 19 returned written informed consent appropriately signed by parents or guardians. The 20 survey was conducted in 2009. Accordingly, 924 youth (522 girls) 11 to 17 years of age 21 had complete data for metabolic variables of interest and were retained for the present 22 analysis. The project was approved by the Scientific Committee of the Federal 23 University of Paraná which requires anonymity and non-transmissibility of data. 
1 Measurements were taken by trained research assistants at each school. Participants

2 wore t-shirts and shorts and shoes were removed. Body height was measured to the 3 nearest $0.1 \mathrm{~cm}$ with a portable stadiometer (Ottoboni HM-210D; RJ, Brazil) and body

4 weight was measured to the nearest $0.1 \mathrm{~kg}$ with a calibrated beam balance scale (Toledo

52096 PP; SP, Brazil). The mean of the two measurements was used for analysis. Waist

6 circumference (WC) was measured at the end of a normal expiration midway between

7 the lower rib margin and iliac crest. Replicate measurements of WC were taken on 89

8 students within the same day. Technical errors of measurement $\left(\sigma_{\mathrm{e}}\right)$ and reliability $(\mathrm{R}){ }^{14}$

$9 \quad$ were $2.09 \mathrm{~cm}$ and 0.97 , respectively.

\section{Blood sampling}

12 Blood samples were collected by trained nurses from the antecubital vein between 8:00 13 and 10:00 am with subjects in a fasted state (10 hours) and seated position. The blood 14 samples were drawn in vacuum tubes gel (Sarstedt). After resting at room temperature 15 for about 30 minutes, samples were centrifuged for 10 minutes at $3000 \mathrm{rpm}$ to obtain 16 serum. Samples were divided into aliquots, separated within 30 minutes and stored at $1780^{\circ} \mathrm{C}$ until analysis. HDL-C, TG, and glucose levels were measured by colorimetric 18 assay on a random access Spectrum CCX analyzer (Abbott Diagnostics, Abbott Park, IL, 19 USA). A single certified laboratory was used for all analyses.

21 Blood pressure (BP)

22 BP was measured according to the method described in The Fourth Report on the 23 Diagnosis, Evaluation, and Treatment of High Blood Pressure in Children and 24 Adolescents ${ }^{15}$. Both systolic blood (SBP) and diastolic blood (DBP) pressures were 25 measured in the right arm using a sphygmomanometer. Two measurements were taken 
1 by trained technicians before blood samples were drawn and after 5 and 10 minutes rest

2 in a seated position. The mean of the two measurements was used for analysis. If the 3 two measurements differed by $>2 \mathrm{mmHg}$, a third measure was obtained, and the mean 4 of the two closest measurements was retained for analysis.

5 Within day technical errors of measurement $\left(\sigma_{\mathrm{e}}\right)$ and reliability $(\mathrm{R}){ }^{14}$ based on 6 replicated measurements of 89 students were as follows: SBP, $2.43 \mathrm{mmHg}$; DBP, 2.52 $7 \mathrm{mmHg}$, while reliability coefficients were as follows: SBP, 0.96; DBP, 0.92.

\section{$9 \quad$ MetS risk score}

10 The definition of the syndrome and cutoff points for specific components vary among 11 studies ${ }^{16}$, but none apply specifically to children and adolescents. Since the primary 12 objective was to investigate the clustering of risk factors relative to MVPA, a 13 continuous metabolic syndrome risk score ${ }^{1,17}$ was used. Each indicator (insulin, 14 triglycerides, blood glucose, HDL-C, BP) was converted to a $\mathrm{Z}$ score, where $Z=$ ([value 15 - mean]/SD). $Z$ scores were multiplied by -1 if necessary to indicate higher metabolic 16 risk with increasing value. $Z$ scores of systolic and diastolic BP were averaged and 17 treated as a single indicator. $\mathrm{Z}$ scores for the five MetS criteria were summed and 18 divided by five to derive an average of clustered metabolic risk score as in other 19 epidemiological studies of youth ${ }^{9,17}$.

\section{Daily physical activity}

22 Each participant completed a dairy protocol ${ }^{18}$ over three complete days (Thursday, 23 Friday and Saturday). The protocol divided each day into 96 periods of 15 minutes.

24 Participants were required to record all activities and to rate the intensity of the primary 25 activity performed in each 15-minute period using a numeric code ranging from one to 
1 nine. Energy expenditure (EE) was estimated from equivalents for each category: [1] 2 sleeping or resting in bed: $0.26 \mathrm{Kcal} / \mathrm{kg} / 15 \mathrm{~min}$; [2] sitting: $0.38 \mathrm{Kcal} / \mathrm{kg} / 15 \mathrm{~min}$; [3] light 3 activity standing: $0.57 \mathrm{Kcal} / \mathrm{kg} / 15 \mathrm{~min}) ;$ [4] slow walking $\simeq 4 \mathrm{~km} / \mathrm{hr}: 0.69$

$4 \mathrm{Kcal} / \mathrm{kg} / 15 \mathrm{~min}$; [5] light manual tasks: $0.84 \mathrm{Kcal} / \mathrm{kg} / 15 \mathrm{~min}$; [6] leisure and recreational

5 sports: $1.2 \mathrm{Kcal} / \mathrm{kg} / 15 \mathrm{~min}$; [7] manual tasks at a moderate pace: $1.4 \mathrm{Kcal} / \mathrm{kg} / 15 \mathrm{~min}$; [8]

6 leisure and sport activities of higher intensity - not competitive: $1.5 \mathrm{Kcal} / \mathrm{kg} / 15 \mathrm{~min}$; [9]

7 very intensive activities - competitive sports: $2.0 \mathrm{Kcal} / \mathrm{kg} / 15 \mathrm{~min}$. Total daily energy

8 expenditure (TDEE) was estimated for each of the three days. Intensity categories 6-9

9 (4.8-7.8 METs) represented MVPA ${ }^{18}$.

10 For inclusion, all (96) 15-min episodes per day had to be completed with a

11 categorical value from 1 to 9 for the three days. Records of participants who did not

12 complete the diary for 3 days were excluded from analysis. Data for 924 youth $(94 \%$ of

13 the initial sample) met the criteria for inclusion and were used for subsequent analyses.

14 There were no significant differences in the distributions of included and excluded 15 participants by $\operatorname{sex}\left[\chi_{(1)}{ }^{2}=0.72 ; \mathrm{p}=0.39\right]$, age $\left[\chi_{(1)}^{2}=6.13 \mathrm{p}=0.41\right]$, and weight status $16\left[\chi_{(1)}^{2}=0.20 \mathrm{p}=0.91\right]$. Data processing and inclusion criteria were the same as in 17 European ${ }^{19,20}$, U.S. ${ }^{21}$ or Asian ${ }^{22}$ studies. Reproducibility of this instrument was $\mathrm{r}=0.91$ 18 in subjects $\geq 10$ years of age ${ }^{18}$ and was validated in adolescents against objective 19 measures of $\mathrm{PA}^{23}$.

\section{Cardiorespiratory fitness (CRF)}

22 CRF was measured with the 20 -meter multistage shuttle run endurance test ${ }^{24}$. The test 23 was scored as the number of "laps" completed at volitional exhaustion. Participants ran 24 between 2 lines, 20 -m apart, following the cadence dictated by a CD emitting audible 
1 signals at prescribed intervals. Initial speed was set at $8.5 \mathrm{~km} / \mathrm{h}$ for the first minute and

2 then was increased $0.5 \mathrm{~km} / \mathrm{h}$ each subsequent minute. When participants could no 3 longer keep up with the pace by reaching the line at the time of the tone, the test was

4 terminated at the second fault and the number of laps completed was recorded. The test 5 provides a valid and reliable field measure of $\mathrm{VO}_{2 \max }$ in adolescents ${ }^{24}$. The protocol 6 was explained in full before the test. All testing was done during physical education 7 classes under dry weather conditions, and carried out and managed by the same 8 researcher who provided the essential instructions for the participants. In addition, two 9 master's level students monitored each line 20-m apart to verify the correct execution of 10 the protocol and also to help encourage and motivate participants to give a maximal 11 effort. At the end of the test, all participants showed signs of intense effort (e.g., 12 hyperpnoea, facial flushing and grimacing, unsteady gait, sweating).

14 Statistical procedures

15 Sex-specific descriptive statistics were calculated for age, height, weight, WC, MVPA, $16 \mathrm{CRF}$, and all metabolic variables. One-way analysis of covariance (ANCOVA) was 17 used to test the effect of gender on the above mentioned variables, controlling for 18 chronological age. All ANCOVAs were followed with Bonferroni-corrected post hoc 19 tests.

20 Prior to analysis, distributions of the clustering of metabolic risk factors, MVPA 21 and CRF scores were tested for normality and normalized if necessary. Insulin, glucose, 22 triglycerides, CRF and MVPA were logarithmically transformed. Log transformation of 23 the variables improved normality for these variables, and as such, they were used as 24 transformed variables in the several analyses. 
1 Adiposity is often indicated as factors affecting habitual PA ${ }^{12,25,26}$ and to a lesser

2 extent metabolic health ${ }^{2}$. Associations between the clustered metabolic risk factors and 3 MVPA, controlling for the potentially confounding effects of chronological age, WC,

4 and CRF were estimated using multiple linear regression analysis. In the minimally 5 adjusted model (Model 1), MVPA was the sole predictor of clustered metabolic risk. 6 WC and chronological age were subsequently added as potential confounders (Model 2). 7 Finally, CRF was then added as a potential confounder (Model 3). Significance was set 8 at 5\%. SPSS 17.0 (SPSS Inc., Chicago, Illinois, USA) was used.

10 RESULTS

11 Characteristics of the sample are summarized in Table 1. About $79 \%$ of boys were 12 categorized as normal weight, $17 \%$ as overweight, and $4 \%$ as obese; corresponding 13 percentages for girls for were $76 \%, 18 \%$, and $6 \%$, respectively. Males and females did 14 not differ in triglycerides, glucose, HDL cholesterol, and diastolic BP. Height, body 15 mass, WC, systolic BP, PA and CRF were, on average, significantly higher in males, 16 whereas insulin level was higher in females. HDL level tended to be higher in females 17 and the difference was marginally significant $(\mathrm{p}=0.056)$.

\section{Bivariate associations}

22 In girls, clustered metabolic risk score was inversely correlated with MVPA $(r=-0.09$, $23 \mathrm{p} \leq 0.05)$ and positively related with weight $(\mathrm{r}=0.36, \mathrm{p} \leq 0.01)$, height $(\mathrm{r}=0.16, \mathrm{p} \leq 0.01)$, 24 and WC $(\mathrm{r}=0.31, \mathrm{p} \leq 0.01)$. MVPA was also positively related to HDL-C ( $\mathrm{r}=0.04$, 
$1 \mathrm{p} \leq 0.05)$ and PA $(\mathrm{r}=0.49, \mathrm{p} \leq 0.01)$, and inversely related to blood pressure $(\mathrm{r}=-0.09$,

$2 \mathrm{p} \leq 0.05)$. The magnitude of those relationships was weak to moderate.

3 Among boys, clustered metabolic risk score was positively correlated with

4 weight $(\mathrm{r}=0.36, \mathrm{p} \leq 0.01)$, height $(\mathrm{r}=0.17, \mathrm{p} \leq 0.01)$, and $\mathrm{WC}(\mathrm{r}=0.42, \mathrm{p} \leq 0.01)$. MVPA was

5 also positively related to PA $(r=0.43, \mathrm{p} \leq 0.01)$ and inversely related to blood pressure

$6(\mathrm{r}=-0.10, \mathrm{p} \leq 0.05)$. The magnitude of the aforementioned relationships was weak to 7 moderate.

[Table 2]

\section{Association between CRF and the clustered metabolic risk score}

12 Results of the regression analyses are summarized in Tables 2 and 3 for females and 13 males, respectively. MVPA was inversely associated with clustered metabolic risk Z14 score after adjustment for several potential confounders in girls $(\beta=-0.08 ; 95 \%$ CI, -0.91 15 to -0.08). In the final model, the additional significant predictors of the clustered 16 metabolic risk were $\mathrm{WC}(\beta=0.55 ; 95 \% \mathrm{CI}, 0.16$ to 0.22$)$, age $(\beta=0.53 ; 95 \% \mathrm{CI}, 0.08$ to $170.30)$ and $\operatorname{CRF}(\beta=-0.06 ; 95 \% \mathrm{CI},-0.04$ to 0.00$)$.

18 In contrast, there was no significantly association between MVPA and the 19 clustered metabolic risk Z-score in boys neither in model $1(\beta=-0.02 ; 95 \% \mathrm{CI},-0.83$ to 20 0.53) nor after adjustment for potential confounding factors (model 3). In the final 21 model, WC ( $\beta=0.61 ; 95 \% \mathrm{CI}, 0.19$ to 0.24$)$, age ( $\beta=0.16 ; 95 \% \mathrm{CI}, 0.12$ to 0.41 ), and 22 CRF ( $\beta=-0.14 ; 95 \%$ CI, -0.03 to -0.01$)$ were the sole significant predictors of clustered 23 metabolic risk in boys. 


\section{DISCUSSION}

3 Research on the MetS and associations with lifestyle behaviours in adolescents is

4 potentially important for identifying subgroups of youth at whom interventions should

5 be targeted. Systematic evaluation of the independent contributions of MVPA to 6 clustered metabolic risk factors in Brazilian youth is lacking. This cross-sectional 7 analysis indicated a negative relationship between the clustering of metabolic risk 8 factors and MVPA in girls 11-17 years, but not in boys. The association in girls was not 9 altered with adjustment for the potential confounding factors included in the regression 10 model. The results for Brazilian adolescent girls were consistent with other studies in 11 showing that overall PA and time spent in MVPA was associated with a healthy 12 cardiometabolic profile in youth ${ }^{2,9,27,28}$.

13 The observed association between MVPA and clustered metabolic risk factors in 14 girls was independent of adiposity and other biological confounding factors such as $15 \mathrm{CRF}$ and chronological age. By inference, it is reasonable to assume that MVPA can 16 improve the metabolic-risk profile of adolescent girls, possibly with the exception of 17 adiposity. Regular PA improves insulin action and glucose transport ${ }^{29}$, and also 18 increases blood flow and oxygen supply through increased capillarization and 19 vasodilatation by nitric oxide, which improves fat metabolism ${ }^{30}$. Regular PA may also 20 affect sympathetic tone with an associated reduction in blood pressure through a more 21 efficient recruitment of the motor units in the muscle ${ }^{31}$. The lack of regular PA was associated with the development of cardiovascular 23 disease risk factors in youth, including lipid disorders, high BP, insulin resistance, and 24 others ${ }^{2,32}$. Data for a representative sample of U.S. adolescents 12-19 years indicated 25 that only about $8 \%$ attained the recommendation of $60 \mathrm{~min} /$ day of PA of moderate or 
Metabolic and behavioral risk factors in youth

1 greater intensity. Similar low rates were also noted in European youth using both

2 objective ${ }^{33}$ and subjective ${ }^{34}$ measures of PA. More recently, only $36 \%$ of Portuguese

3 youth $10-11$ years (boys $=51.6 \%$, girls $=22.5 \%$ ) and $4 \%$ of youth $16-17$ years

4 (boys $=7.9 \%$, girls $=1.2 \%$ ) were considered sufficiently active by achieving 60 minutes

5 of MVPA daily ${ }^{35}$. The majority (58\%) of Brazilian adolescent girls in the present study

6 did not achieve 60 minutes of MVPA daily; corresponding data for boys was 30\%.

7 Overall, evidence from many parts of the world suggests significant metabolic health

8 risk among youth which has implication for public health as metabolic risk and PA tend

9 to track from adolescence into adulthood ${ }^{36}$.

10 Our results from boys contradict findings of girls suggesting that MVPA is not

11 significantly associated with the clustered of metabolic outcomes. Those sex-differences

12 can be explained, in part, by the between-individual variability of PA which vary

13 between boys and girls; this variability impacts the ability to measure MVPA and

14 consequently influences their relationship with metabolic outcomes. When interpreting

15 those sex-related differences, the significant difference in the prevalence of some

16 outcomes of MetS between boys and girls should be also considered, such as insulin and

17 systolic BP of the present study; this may be related to hormonal differences, such as

18 testosterone and sex-hormone binding globulin between genders ${ }^{37}$. Those notable

19 differences between genders, for which probably we do not have an adequate

20 explanation, could possibly be explained by more focus of public educational programs

21 on girls compared to boys or even by some specific cultural and lifestyle differences,

22 leading to discrepant trend on the association between the clustered of metabolic risk

23 factors and PA in boys and girls.

24 Some observational studies ${ }^{10,28,38}$ examined large and heterogeneous samples of

25 children and adolescents, suggesting that the findings are quite generalizable to the 
1 general population. However, in some cross-sectional studies that were employed self-

2 reported measures of $\mathrm{PA}$, the reported associations with the MetS were either weak or 3 modest in strength, and were non-significant ${ }^{39,40}$. In contrast, studies that used objective 4 measures (e.g. accelerometers) to assess PA ${ }^{10}$, it was reported strong and significant 5 relations with the MetS. Thus, variation in results among studies may be related to the 6 assessment of PA and its multidimensional nature per see; PA protocols may be affected 7 by the nature of behavior recalled. Most daily activities are intermittent and may involve 8 substantial rest periods, which may lead to significant overestimation of time spent on 9 daily activities ${ }^{41}$. Such intermittent activities are probably more difficult to define or 10 quantify than occupational activities or structured exercises ${ }^{42}$. Therefore, it is plausible

11 that the lack of observed associations between MVPA and clustered metabolic risk in 12 boys were likely to be due, in part, to measurement accuracy since self-report 13 instrument are often viewed as having less precision for high intensity levels of PA ${ }^{42,43}$.

14 However, despite of some studies have revealed clear associations, the nature (e.g., 15 linear or curvilinear) of the dose-response relation is still unclear claiming for further 16 research with different analytical approach and design.

17 Additional sources of variation among studies, in the association of MVPA and 18 the clustering of metabolic risk factors, may be related to the cut-off criteria used to 19 define MetS and in turn the metabolic risk factors in the present study. Results for the 20 Brazilian adolescents showed considerable variation in prevalence of MetS. For 21 example, only $1.5 \%$ of the female adolescents from the Vitoria region of Brazil were 22 classified as having $\geq 3 \mathrm{MRF}^{44}$ compared to $6.5 \%$ of girls from Curitiba in the present 23 study. On the other hand, $13.7 \%$ and $15 \%$ of adolescents from the São Paulo region ${ }^{45}$ 24 were classified as having MetS according to different criteria ${ }^{47}$. Results of the different 25 surveys of Brazilian youth should thus be evaluated and interpreted with care. Further 
Metabolic and behavioral risk factors in youth

1 efforts are needed to encourage standardization of criteria for MetS in children and 2 adolescents.

3 In summary, generalization of the observed association between MVPA and the

4 clustered metabolic risk factors in Brazilian youth to other populations of adolescents

5 should be done with care. The study has several limitations that should be noted. First,

6 causal relationship between low MVPA and increased risk for MetS cannot be inferred

7 from a cross-sectional design. Second, an indicator of biological maturity status was not

8 included in the study protocol. Although chronological age was adjusted for the

9 analyses, this may not be sufficient because biological maturity different may impact in

10 insulin levels of youth and therefore should be considered in future research. Third, the

11 results are based on a relatively small sample of Brazilian girls 11 to 17 years living in

12 the urban center of the Paraná region. Further, the use of PA self-report instruments is

13 challenging and, as aforementioned, requires several cautions and standardizing

14 procedures to decrease potential measurement errors. Although different models of

15 association had been tested, experimental and longitudinal investigations are needed to

16 draw conclusions about the etiologic influence of PA, WC and fitness on

17 cardiometabolic risk.

19 CONCLUSION

20 MVPA was independently associated with an increased risk of MetS in Brazilian 21 adolescent girls, but not in boys. Findings highlight the importance of preventive 22 actions against metabolic risk in female youth which may need to target active lifestyle. 23 Additional efforts are needed to encourage research with different analytical approach 24 and standardization of criteria for MetS in children and adolescents. 
1 Acknowledgment

2 The authors also acknowledge the support provided by the CAPES (Coordenação de 3 Aperfeiçoamento de Pessoal de Nível Superior) - "Impacto do peso ao nascimento e do 4 estilo de vida sobre fatores de risco metabólico, hiperandrogenismo e anovulação em 5 meninas e adolescentes" Project.

7 Declaration of interest: The authors report no conflicts of interest. The authors alone 8 are responsible for the content and writing of the paper.

\section{REFERENCES}

11 1. Eisenmann, J.C., Laurson, K.R., DuBose, K.D., Smith, B.K. \& Donnelly, J.E.

12 Construct validity of a continuous metabolic syndrome score in children.

13 Diabetol Metab Syndr 2, 8 (2010).

14 2. Steele, R.M., Brage, S., Corder, K., Wareham, N.J. \& Ekelund, U. Physical

15 activity, cardiorespiratory fitness, and the metabolic syndrome in youth. $J$ Appl

$16 \quad$ Physiol 105, 342-351 (2008).

17 3. Cook, S., Weitzman, M., Auinger, P., Nguyen, M. \& Dietz, W.H. Prevalence of

18 a metabolic syndrome phenotype in adolescents: findings from the third

19 National Health and Nutrition Examination Survey, 1988-1994. Arch Pediatr

$20 \quad$ Adolesc Med 157, 821-827 (2003).

21 4. Lopez-Capape, M., et al. Frequency of the metabolic syndrome in obese Spanish pediatric population. Eur J Endocrinol 155, 313-319 (2006).

23 5. Dhuper, S., et al. Utility of the modified ATP III defined metabolic syndrome and severe obesity as predictors of insulin resistance in overweight children and adolescents: a cross-sectional study. Cardiovasc Diabetol 6, 4 (2007). 
Metabolic and behavioral risk factors in youth

$16 . \quad$ Nasreddine, L., et al. Obesity is associated with insulin resistance and components of the metabolic syndrome in Lebanese adolescents. Ann Hum Biol 39, 122-128 (2012).

47 Cumming SP \& C, R. Physical activity, physical fitness and health: current concepts. in Paediatric Exercise Science and Medicine (ed. Armstrong, N.) 327338 (Oxford University Press, Oxford, 2008).

7 8. Reichert, F.F., Baptista Menezes, A.M., Wells, J.C., Carvalho Dumith, S. \& Hallal, P.C. Physical activity as a predictor of adolescent body fatness: a

9. Brage, S., et al. Features of the metabolic syndrome are associated with objectively measured physical activity and fitness in Danish children: the European Youth Heart Study (EYHS). Diabetes Care 27, 2141-2148 (2004).

10. Andersen, L.B., et al. Physical activity and clustered cardiovascular risk in children: a cross-sectional study (The European Youth Heart Study). Lancet 368, 299-304 (2006).

11. Holmes, M.E., Eisenmann, J.C., Ekkekakis, P. \& Gentile, D. Physical activity, stress, and metabolic risk score in 8- to 18-year-old boys. J Phys Act Health 5, 294-307 (2008).

12. Malina, R.M., Bouchard, C. \& Bar-Or, O. Growth, Maturation, and Physical Activity, (Human Kinetics, Champaign, IL, 2004).

13. Hallal, P.C., et al. Global physical activity levels: surveillance progress, pitfalls, and prospects. Lancet 380, 247-257 (2012).

14. Mueller, W.H. \& Martorell, R. Reliability and accuracy of measurement. in Anthropometric standardization reference manual (ed. T. G. Lohman, A.F.R., \& R. Martorell) 83 -86 (Human Kinetics, Champaign, Illinois, 1988). 
1 15. National High Blood Pressure Education Program. The fourth report on the diagnosis, evaluation, and treatment of high blood pressure in children and adolescents. Pediatrics 114, 555-576 (2004).

16. Cavali, M.E., Brasileiro, R. \& Taddei, J. Síndrome metabólica: comparação de critérios diagnósticos. Jornal de Pediatria 86, 325-330 (2010).

17. Eisenmann, J.C. On the use of a continuous metabolic syndrome score in pediatric research. Cardiovasc Diabetol 7, 17 (2008).

18. Bouchard, C., et al. A method to assess energy expenditure in children and adults. Am J Clin Nutr 37, 461-467 (1983).

19. Machado-Rodrigues, A.M., et al. Agreement in activity energy expenditure assessed by accelerometer and self-report in adolescents: variation by sex, age, and weight status. J Sports Sci 29, 1503-1514 (2011).

20. Machado-Rodrigues, A.M., et al. Physical activity and energy expenditure in adolescent male sport participants and nonparticipants aged 13 to 16 years. $J$ Phys Act Health 9, 626-633 (2012).

21. Katzmarzyk, P.T., Malina, R.M., Song, T.M. \& Bouchard, C. Physical activity and health-related fitness in youth: a multivariate analysis. Med Sci Sports Exerc 30, 709-714 (1998).

22. Huang, Y.C. \& Malina, R.M. Physical activity and health-related physical fitness in Taiwanese adolescents. Journal of physiological anthropology and applied human science 21, 11-19 (2002).

23. Machado-Rodrigues, A.M., et al. Concurrent validation of estimated activity energy expenditure using a 3-day diary and accelerometry in adolescents. Scand J Med Sci Sports 22, 259-264 (2012). 
1 24. Leger, L.A., Mercier, D., Gadoury, C. \& Lambert, J. The multistage 20 metre shuttle run test for aerobic fitness. $J$ Sports Sci 6, 93-101 (1988).

3 25. Freitas, A., et al. Crescimento somático, maturação biológica, aptidão física, actividade física e estatuto socioeconómico de crianças e adolescentes madeirenses, (Universidade da Madeira, Funchal, 2002).

$626 . \quad$ Jimenez-Pavon, D., et al. Influence of socioeconomic factors on fitness and fatness in Spanish adolescents: The AVENA study. Int J Pediatr Obes (2010).

8 27. Hsu, Y.W., et al. Physical activity, sedentary behavior, and the metabolic syndrome in minority youth. Med Sci Sports Exerc 43, 2307-2313 (2011).

28. Rizzo, N.S., Ruiz, J.R., Hurtig-Wennlof, A., Ortega, F.B. \& Sjostrom, M. Relationship of physical activity, fitness, and fatness with clustered metabolic risk in children and adolescents: the European youth heart study. J Pediatr 150,

30. Kraus, W.E., et al. Effects of the amount and intensity of exercise on plasma lipoproteins. N Engl J Med 347, 1483-1492 (2002).

31. Ramsay, J.A., et al. Strength training effects in prepubescent boys. Med Sci Sports Exerc 22, 605-614 (1990).

20 32. Ortega, F.B., Ruiz, J.R., Hurtig-Wennlof, A. \& Sjostrom, M. [Physically active adolescents are more likely to have a healthier cardiovascular fitness level independently of their adiposity status. The European youth heart study]. Rev Esp Cardiol 61, 123-129 (2008). 
$133 . \quad$ Ruiz, J.R., et al. Objectively measured physical activity and sedentary time in European adolescents: the HELENA study. Am J Epidemiol 174, 173-184 (2011).

34. Tammelin, T., Ekelund, U., Remes, J. \& Nayha, S. Physical activity and sedentary behaviors among Finnish youth. Med Sci Sports Exerc 39, 1067-1074 (2007).

7 35. Baptista, F., et al. Prevalence of the Portuguese population attaining sufficient physical activity. Med Sci Sports Exerc 44, 466-473 (2012).

9 36. Kjonniksen, L., Anderssen, N. \& Wold, B. Organized youth sport as a predictor of physical activity in adulthood. Scand J Med Sci Sports 19, 646-654 (2009).

37. Agirbasli, M., et al. Sex hormones and metabolic syndrome in children and adolescents. Metabolism 58, 1256-1262 (2009).

38. Ekelund, U., et al. Moderate to vigorous physical activity and sedentary time and cardiometabolic risk factors in children and adolescents. JAMA 307, 704712 (2012).

39. Ramirez-Lopez, G., et al. Weight, physical activity, and smoking as determinants of insulinemia in adolescents. Archives of medical research 32, 208-213 (2001).

40. Kelishadi, R., et al. Association of physical activity and the metabolic syndrome in children and adolescents: CASPIAN Study. Horm Res 67, 46-52 (2007).

41. Slootmaker, S.M., Schuit, A.J., Chinapaw, M.J., Seidell, J.C. \& van Mechelen, W. Disagreement in physical activity assessed by accelerometer and self-report in subgroups of age, gender, education and weight status. Int J Behav Nutr Phys Act 6, 17 (2009). 
Metabolic and behavioral risk factors in youth

1 42. Armstrong, N. \& Welsman, J.R. The physical activity patterns of European youth with reference to methods of assessment. Sports Med 36, 1067-1086 (2006).

4 43. Machado Rodrigues, A.M. Energy expenditure, physical activity and sedentary behaviour, (Coimbra, 2013).

6 44. Rodrigues, A.N., et al. Cardiovascular risk factors, their associations and presence of metabolic syndrome in adolescents. $J$ Pediatr (Rio J) 85, 55-60 (2009).

9 45. Cavali Mde, L., Escrivao, M.A., Brasileiro, R.S. \& Taddei, J.A. Metabolic syndrome: comparison of diagnosis criteria. J Pediatr (Rio J) 86, 325-330 (2010). 
2 Table 1. Descriptive characteristics of participants.

3

Variable

All Boys

Girls

$(\mathrm{n}=402) \quad(\mathrm{n}=522)$

Chronological age, years

$13.7 \pm 1.9$

$13.9 \pm 1.9$

$13.6 \pm 1.9$

Weight (kg)

$52.2 \pm 13.0$

$54.5 \pm 14.5$

$50.5 \pm 11.3 * *$

Height (m)

$159.0 \pm 11.2$

$162.8 \pm 13.4$

$156.0 \pm 8.1 * *$

$\mathrm{WC}(\mathrm{cm})$

$68.2 \pm 8.8$

$70.3 \pm 9.1$

$66.5 \pm 8.2 * *$

Insulin $^{\mathrm{a}}(\mathrm{pmol} / \mathrm{l})$

$6.03 \pm 3.78$

$5.02 \pm 4.18$

$6.80 \pm 3.25 * *$

Glucose $^{\mathrm{a}}(\mathrm{mmol} / \mathrm{l})$

$94.64 \pm 12.08$

$94.98 \pm 10.69$

$94.38 \pm 13.05$

Triglycerides ${ }^{\mathrm{a}}$ (mmol/1)

$84.98 \pm 35.10$

$84.17 \pm 39.74$

$85.61 \pm 31.08$

HDL cholesterol (mmol/l)

$44.79 \pm 10.00$

$44.00 \pm 9.26$

$45.39 \pm 10.51$

Systolic BP (mmHg)

$102.33 \pm 12.41$

$104.32 \pm 12.50$

$100.80 \pm 12.13 *$

Diastolic BP (mmHg)

$67.97 \pm 9.94$

$68.76 \pm 10.03$

$67.36 \pm 9.84$

Metabolic syndrome ( $Z$ score)

$0.00 \pm 3.02$

$-0.1 \pm 3.17$

$0.09 \pm 2.90$

$\mathrm{CRF}^{\mathrm{a}, \mathrm{b}}(\#)$

MVPA $^{\text {a }}$ (minutes)
$40.9 \pm 21.0$

$77.2 \pm 80.2$
$56.2 \pm 21.6$

$102.8 \pm 80.9$ 
1 Table 2. Prediction of the metabolic syndrome $\mathrm{Z}$ score in females aged 11-17 years.

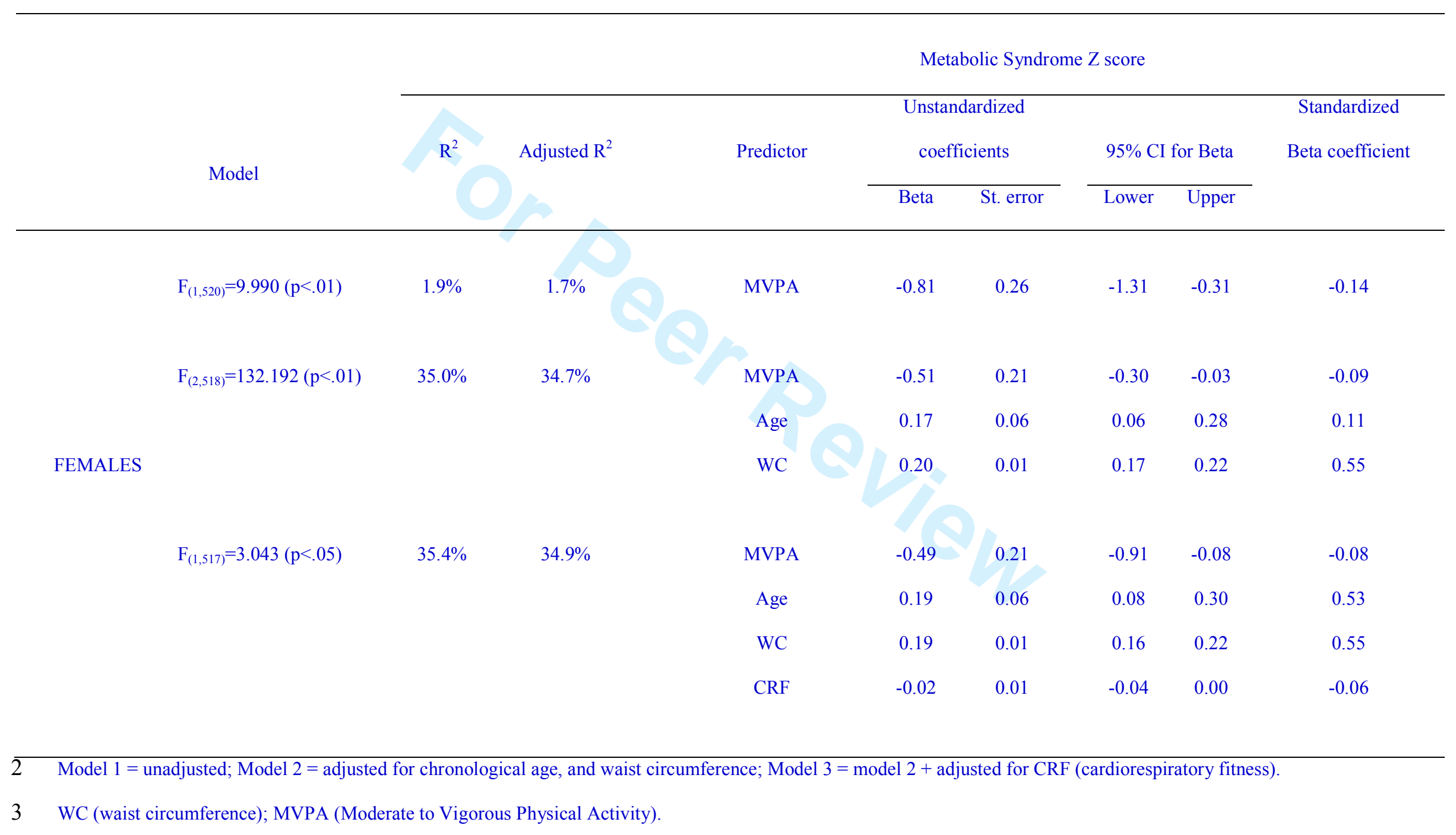


1 Table 3. Prediction of the metabolic syndrome $\mathrm{Z}$ score in males aged 11-17 years.

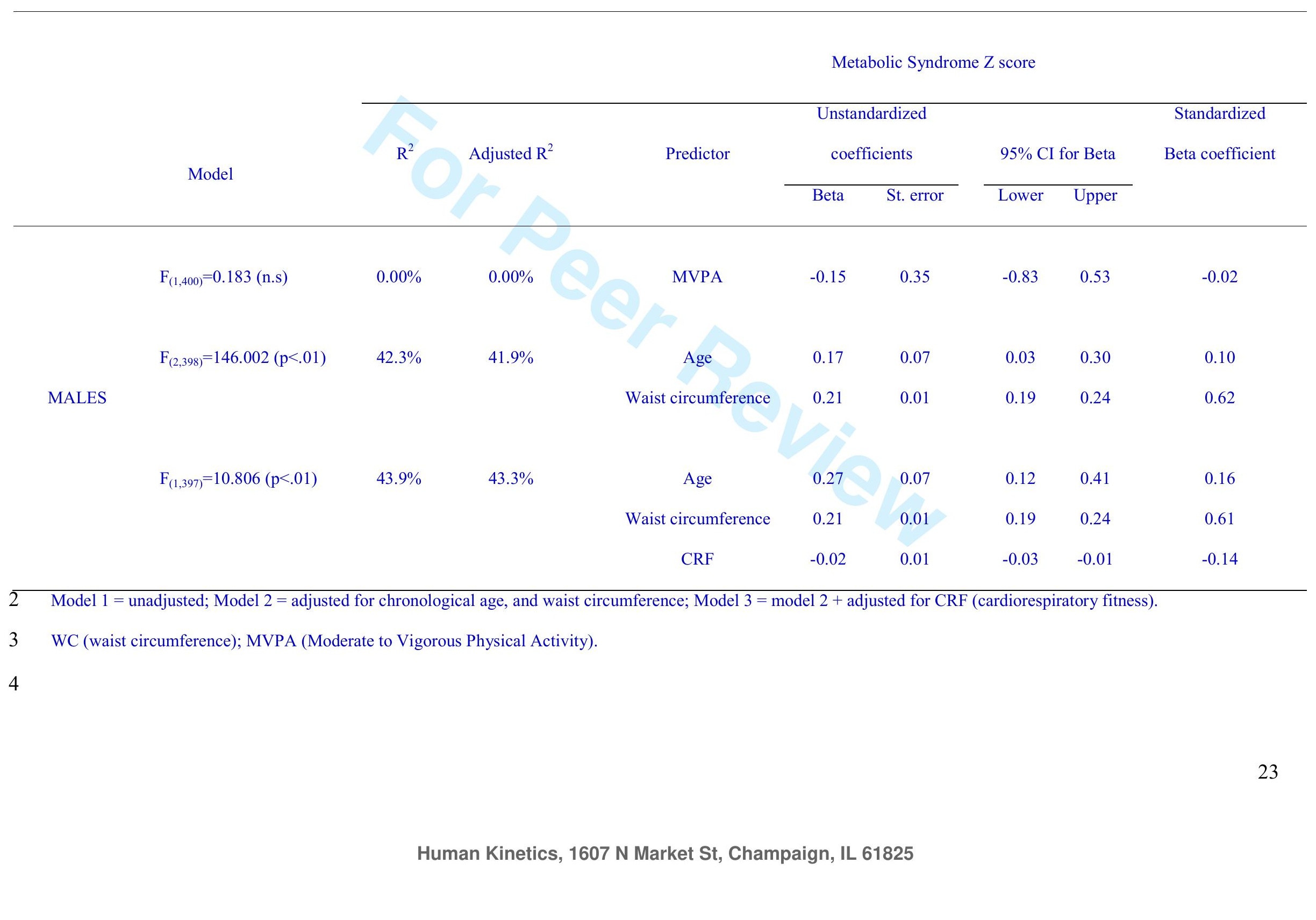

